\title{
The Effect of Mesobotox Together with Intense Pulsed Light on Facial Wrinkles and Erythema
}

\author{
Ahmed El Bedewi \\ Dermatology Unit, National Center for Radiation Research and Technology, Cairo, Egypt. \\ Email: aelbedewi@gmail.com
}

Received December $16^{\text {th }}, 2011$; revised January $4^{\text {th }}, 2012$; accepted January $15^{\text {th }}, 2012$

\begin{abstract}
Facial ageing is a gradual process which could be due to intrinsic and extrinsic causes, it ultimately results in the appearance of gravity-induced tissue ptosis, wrinkles, epidermal and dermal atrophy, dryness, senile lentigo, flushing, telangiectasia, and enlarged pores. Mesobotox treatment is a technique of microinjections of diluted Botulinum toxin type A (BTX) to preserve the facial mobility in order to preserve the natural beauty. BTX treatment enhances the aesthetic improvements attained with IPL. Ten patients of age ranging between 40 - 60 years old (mean $45 \pm 10.3$ years) with skin type ranging between (III - V) were treated using BTX and Intense Pulsed Light (IPL) using cutoff filter 550 $\mathrm{nm}$ with a fluence of $25 \mathrm{~J} / \mathrm{cm}^{2}$ together as a combination therapy. All patients received standard IPL treatment and were assigned to receive eight $0.1 \mathrm{~mL}$ intradermal injections of BTX in each cheek ( $8 \mathrm{U}$ total dose). Vertical lines within the forehead also received five $0.1 \mathrm{~mL}$ intradermal injections of BTX. Small wrinkles and fine lines, erythema, apparent pore size, skin texture, and overall appearance were evaluated after one week. A significantly higher proportion of patients showed improvement in small wrinkles and erythema with IPL plus BTX compared with the baseline demonstrated by computerized image analysis. In conclusion, A combined therapy of mesobotx and IPL is an effective and safe treatment for fine wrinkles, telangiectasia, flushing.
\end{abstract}

Keywords: Ageing; Skin; Intense Pulsed Light; Botulinum Toxin Type A; BTX; Rejuvenation; Erythema; Wrinkles; Telangiectasia

\section{Introduction}

As life expectancy has prolonged nowadays, interest has increased to change patterns of life, which include improving the consequences of skin ageing through slowing the ageing process. There are two main process of skin ageing either intrinsic or extrinsic. Intrinsic ageing reflects genetic background in which skin appears thin with exaggerated expression lines. However, extrinsic ageing is caused by external factors such as smoking, excessive use of alcohol, poor nutrition and sun exposure. Extrinsically aged skin appears predominately not only by wrinkles, but also by pigmented lesions such as freckles, lentigines and patchy hyperpigmentation, and depigmented lesions such as guttate hypomelanosis. Epidermal and dermal atrophy, rough skin texture, flushing, erythema, telangiectasias, dilated pores and loss of tone and elasticity are also other signs of skin aging [1].

Botulinum toxin type A (BTX) has been used for more than a decade in facial aesthetics for the treatment of dynamic rhytids. Mesobotox involves microinjections of very small doses of botox that are disseminated all over the face to reduce fine wrinkles without affecting the facial ability of expressing mimic gestures and emotions. Also, it improves the surface of the skin and affects sebaceous glands through diminishing the size of dilated pores. Over the past few years, the use of BTX has broadened and it is now commonly used as an adjunct to enhance or sustain other rejuvenation procedures, both ablative and nonablative. Pretreatment with BTX has been shown to enhance the effects of laser resurfacing on the upper face [2]. Similarly, pretreatment of glabellar lines with a hyaluronic acid filler has been shown to enhance the effects of BTX [3]. Intense pulsed light (IPL) is a nonablative light-based treatment whose safety and efficacy profile has made it a first-line treatment for hair removal, vascular lesions and signs of photoaging. The results of previous studies demonstrate that IPL alone can also improve the appearance of facial erythema and rhytids [4-8]. In 2008 Khoury and her coworkers studied IPL and BTX on 14 females on a double-blind, split-face study. They concluded that the adjunctive use of BTX enhances the improvement in small wrinkles and fine lines, and possibly erythema, achieved with IPL alone [9]. 


\section{Material and Method}

This study was carried out on ten Egyptian female patients with age ranging from 40 - 60 years with mean $(45$ \pm 10.3 ) years, skin type ranging between III - V. Patients were not allowed to have had microdermabrasion, light skin peels, nonablative laser, light, or radiofrequency, or deep skin peels, ablative laser treatment, BTX treatment, filler treatment, or cosmetic surgery in the preceding 3 months. Patients were ineligible for the study if they were photosensitive, pregnant, or had hemophilia, viral hepatitis, cancer, any immunocompromising disease, or any neurological condition. They were also ineligible if they had any scarring, tattoos, or hemangiomas that could interfere with treatment or were using over-thecounter glycolic acid products, abrasive products, topical antibiotics, oral isotretinoin, or any medication that can cause photosensitivity.

\subsection{Treatment Protocol}

Third generation IPL (Medical Bio Care, Sweden) with filtered light to selectively target the melanin, hemoglobin and collagen by selective photo-thermolysis was used. A sapphire crystal wave-guide conducts the filtered light to the target chromophores in the tissues. It operates through creation a direct incoherent light beam of spectrally balanced bandwidth. All patients were treated fullface treatments with IPL. The energy density was delivered was $25 \mathrm{~J} / \mathrm{cm}^{2}$ with pulse duration $50-70 \mathrm{~ms}$, delivered by cut-off filter $(550 \mathrm{~nm})$ with triple pulse illumination. Each treatment was given by applying a thin layer of cold optical contact transparent gel (SonogelGermany) over the area to be treated. Energy was delivered through a $10 \times 20 \mathrm{~mm}$ light guide that was precooled by ice water or cryogen spray. No pre-anesthetic preparations were used. IPL treatment was followed by BTX (Allergan-USA) injections in each cheek. Injecting BTX after IPL avoids the potential for blood contaminating from the IPL crystal. After the IPL treatment, the patient's face was cleansed and then wiped with an alcohol pad. The physician then injected each cheek with a total of eight injections of their assigned treatment. 0.1 $\mathrm{mL}$ of BTX (1U of BTX per injection for a total dose of $8 \mathrm{U}$, as the vial was reconstituted with $10 \mathrm{~mL}$ preservative-free sterile saline). The injections were performed in a pattern across the face at approximately $2-\mathrm{cm}$ intervals. Each injection was made using a 30-gauge tuberculin syringe and was carefully placed into the skin at a $45^{\circ}$ angle to create an intradermal wheal similar to a tuberculin test injection. Great care was taken to inject superficially rather than intramuscularly and to avoid cheek bone and maseeter muscle. After treatment, patients were advised to protect their skin from the sun using sun- screen.

\subsection{Evaluation}

Skin displacement analysis Horizontal skin displacement due to maximum contraction of the fronto-glabellar area was analyzed at Day 0 immediately before the injection of BTX, and at Day 7 post-injection. Vertical and horizontal black lines cross at one sample point of interest above the right eyebrow (X0, Y0). The same 2 points are marked during maximum fronto-glabellar contraction which serves as the fixed-point of the geometric system, thereby allowing shifts or movements of the face at other points to be calculated from a standardized Reference point. The horizontal shift of the point of interest above the eyebrow during maximum fronto-glabellar contraction (X1 contraction) compared with its position under relaxed conditions (X2) forms the basis of numeric calculation. The amplitude of the horizontal shift from the relaxed to the contracted situation was calculated as follows:

$$
\Delta \mathrm{X}=[(\mathrm{X} 2 \text { contraction }-\mathrm{X} 0)-(\mathrm{X} 1 \text { relaxed }-\mathrm{X} 0)]
$$

where $\Delta \mathrm{X}$ is given in the dimension of pixels in the digital photograph. To obtain movement amplitudes in millimeters, the value of $\Delta \mathrm{X}$ is normalized for the number of pixels along the length of each $5 \mathrm{~mm}$ box in each digital photograph. Digital photographs were taken at Day 0, before injection of BTX, and 7 post-injection. At each time-point, 3 cycles of relaxed muscle tension and maximum contraction were captured [10]. Standardized facial photographs (frontal view) were taken of each patient using computerized Image analysis, the data were obtained using Leica Qwin 500 image analyzer computer system (England). The image analyzer consisted of a colored video camera, colored monitor, hard disc of IBM personal computer and controlled by Leica Qwin 500 software. The image analyzer was first calibrated automatically to convert the measurement units (pixels) produced by the image analyzer program into actual micrometer units. Statistical significance between groups for each parameter was determined using Student t-test using SPSS program.

\section{Results}

All patients showed at least mild improvement from baseline in small wrinkles and fine lines which was significantly greater after IPL-BTX treatment in relation to the base line depending on the skin type. Mild improvement in erythema was observed in $100 \%$ at day 7 (Table 1 and Figure 1). Also, this treatment regimen was associated with generally comparable improvement in apparent pore size, skin texture, skin roughness, and overall appearance. 
In the other hand, when we compared between the average reduction of maximum frontoglabellar contraction elicited skin displacement amplitudes after BTX and IPL treatment. It was found that after 7 days the percentage of contraction was reduced markedly in a highly significant manner as shown in Table 2.

\section{Discussion}

The use of IPL plus BTX regimen resulted in a better clinical improvement especially small wrinkles and fine lines despite the small number of patients used in this study. The intradermal injection of BTX has been reported previously in the axilla for the treatment of hyperhidrosis [11]. Even though the injections were placed superficially within the face in this study and the volume

Table 1. Shows response of different skin types after 7 days of IPL and BTX treatment.

\begin{tabular}{ccccc}
\hline Skin type & Erythema & Fine wrinkles & Telangectasia & Pore Size \\
\hline III & +++ & +++ & + & + \\
IV & +++ & +++ & + & + \\
V & ++ & ++ & + & + \\
\hline
\end{tabular}

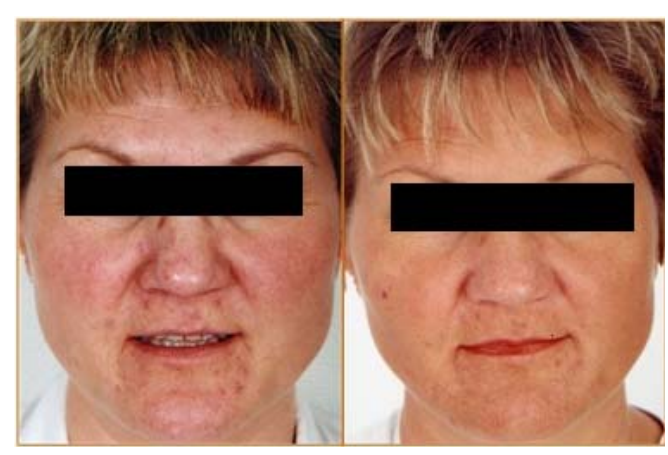

(a)

(b)

Figure 1. Improvement in erythema and wrinkles after treatment with IPL plus BTX. (a) Pretreatment with relaxed fronto-glabellar area and erythema in the cheeks and chin; (b) After treatment with IPL plus BTX, erythema and wrinkles are diminished.

Table 2. Shows time course of average reduction of maximum frontoglabellar contraction elicited skin displacement amplitudes, normalized to Day 0 prior to BTX injection.

\begin{tabular}{cccc}
$\begin{array}{c}\text { Above eye brow } \\
\text { (Lateral to midline) }\end{array}$ & Day 0 & Day 7 & Student t-test \\
\hline $20 \mathrm{~mm}$ & $75 \% \pm 11 \%$ & $12 \% \pm 15 \%$ & $<0.001$ \\
$30 \mathrm{~mm}$ & $83 \% \pm 20 \%$ & $15 \% \pm 186 \%$ & $<0.001$ \\
$40 \mathrm{~mm}$ & $82 \% \pm 17 \%$ & $15 \% \pm 16 \%$ & $<0.001$ \\
\hline
\end{tabular}

Student t-test: $<0.001=$ highly significant, $<0.05=$ significant . of injection minimized at $0.1 \mathrm{~mL}$ to reduce the potential for diffusion [12], it is possible that improvement of wrinkles in the periocular area may have been at least partly attributable to the migration of BTX from its site of injection.

Migration of BTX might also have the potential to weaken the activity of zygomatic muscles. However, none of our ten patients reported facial asymmetry or any loss of muscle function or any side effects. There are other reports in the literature showing improvement of facial, neck, and chest wall erythema and flushing with intradermal injections of botulinum toxin. Moreover, in 2004 Carruthers and Carruthers showed a greater degree of improvement in telangiectasia with IPL plus BTX than IPL alone. The mechanism behind any improvement in erythema is not fully understood but has been partially elucidated [13]. Therefore, it is possible that the improvement in erythema may be due to BTX mediated inhibition of the release of vasodilating neuropeptides.

Non-ablative IPL rejuvenation represents the one of the recent approaches to improve photo-damaged skin. Dermal remodeling is thought to occur through increased collagen I deposition with collagen re-organization into parallel arrays of compact fibrils, which results in clinical improvement. However, the biological mechanism of selective photo-thermal collagen stimulation is not yet fully explained [8].

In the present study, we evaluated the efficacy and safety of a non-coherent intense pulsed light in the nonablative rejuvenation together with BTX. A satisfactory improvement was noticed on telangiectasias, flushing, fine wrinkles, dilated pores and laxity. Single treatment gave considerably good results; but repeated treatments after 3 - 4 weeks may results in visible improvement of wrinkles and all elements of skin photo-damage. In conclusion: although, the variance in skin type and degree of photo ageing; IPL together with mesobotox could be a treatment of choice in facial rejuvenation with satisfactory results. The use of a new method for assessing skin movements elicited by muscles inserting the overlaying skin. Skin displacement analysis demonstrated high accuracy within a few millimeters when evaluating IPL together with BTX-induced relaxation in the fronto-glabellar area. Although, we recommend further comparative study between IPL plus BTX and IPL alone, in order to prove if the latter has any additive effect to Botulinum toxin in the treatment of wrinkles.

\section{REFERENCES}

[1] L. Baumann, "Cosmetic Dermatology; Principles and Practice,” McGraw-Hill Companies, New York, 2002.

[2] M. S. Zimbler, J. B. Holds, M. S. Kokoska, et al., "Effect of Botulinum Toxin Pretreatment on Laser Resurfacing Results: A Prospective, Randomized, Blinded Trial," Ar- 
chives of Facial Plastic Surgery, Vol. 3, No. 3, 2001, pp. 165-169. doi:10.1001/archfaci.3.3.165

[3] J. Carruthers and A. Carruthers, "A Prospective, Randomized, Parallel Group Study Analyzing the Effect of BTX-A and Nonanimal Sourced Hyaluronic Acid (NASHA, Restylane) in Combination Compared with NASHA (Restylane) Alone in Severe Glabellar Rhytides with a Hyaluronic Acid Derivative Compared with the Derivative and BTXA," Dermatologic Surgery, Vol. 29, No. 8, 2003, pp. 802-809. doi:10.1046/j.1524-4725.2003.29212.x

[4] A. El-Bedewi, "Broad Spectrum Intense Pulsed Light Source (IPL)-A New Therapeutic Modality in the Coming Millennium," Dermatology, Vol. 7, No. 2, 2000, p. 22.

[5] A. El-Bedewi, "Hair Removal with Intense Pulsed Light," Lasers in Medical Science, Vol. 19, No. 1, 2004, pp. 48-51.

[6] M. P. Goldman, R. A. Weiss and M. A. Weiss, "Intense Pulsed Light as a Nonablative Approach to Photoaging," Dermatologic Surgery, Vol. 31, Suppl. 3, 2005, pp. 11791187. doi:10.1111/j.1524-4725.2005.31924

[7] D. J. Goldberg and K. B. Cutler, "Nonablative Treatment of Rhytids with Intense Pulsed Light," Lasers in Surgery and Medicine, Vol. 26, No. 2, 2000, pp. 196-200. doi:10.1002/(SICI)1096-9101(2000)26:2<196::AID-LSM $\underline{10>3.0 . \mathrm{CO} ; 2-9}$
[8] A. El-Bedewi, "Intense Pulsed Light in Dermatology," 4th World Congress of the International Academy of Cosmetic Dermatology 2005 (IVth IACD), Paris.

[9] J. G. Khoury, R. Saluja and M. P. Goldman, “The Effect of Botulinum Toxin Type A on Full-Face Intense Pulsed Light Treatment: A Randomized, Double-Blind, SplitFace Study," Dermatologic Surgery, Vol. 34, No. 8, 2008, pp. 1062-1069. doi:10.1111/j.1524-4725.2008.34207.x

[10] T. Proebstle, "Skin Displacement Analysis (SDA): A Tool for the Quantitative Evaluation of Skin Movements Elicited by Underlying Muscles in the Face and Neck Area," Clinical, Cosmetic and Investigational Dermatology, Vol. 4, 2011, pp. 27-33. doi:10.2147/CCID.S18185

[11] M. Heckmann, S. Breit, A. Ceballos-Baumann, et al., "Side-Controlled Intradermal Injection of Botulinum Toxin A in Recalcitrant Axillary Hyperhidrosis," Journal of American Academy Dermatology, Vol. 41, No. 6, 1999, pp. 987-990. doi:10.1016/S0190-9622(99)70258-6

[12] S. K. Kranendonk, L. K. Ferris and S. Obagi, "Re: Botulinum Toxin for the Treatment of Facial Flushing," Dermatologic Surgery, Vol. 31, 2005, p. 491.

[13] J. Carruthers and A. Carruthers, "The Effect of Full-Face Broadband Light Treatments Alone and in Combination with Bilateral Crow's Feet Botulinum Toxin Type A Chemodenervation," Dermatologic Surgery, Vol. 30, No. 3, 2004, pp. 355-366. doi:10.1111/j.1524-4725.2004.30101.x 